Pacific Journal of Mathematics

ON A CONJECTURE OF K. MURASUGI 


\title{
ON A CONJECTURE OF K. MURASUGI
}

\author{
William S. MASSEY AND LoRENZo Traldi
}

\begin{abstract}
K. Murasugi has conjectured that if every pair of the $\mu$ components of a classical link $L$ has linking number \pm 1 , then the group $G$ of $L$ will have the property that $G_{q} / G_{q+1} \cong F_{q} / F_{q+1} \forall q \geq 2$, where $F$ is free on $\mu-1$ generators. (The conjecture has been verified by other authors.) Here we show that this property is equivalent to the special case $G_{2} / G_{3} \cong F_{2} / F_{3}$, and also give other equivalent conditions.
\end{abstract}

1. Introduction. A (tame) link in the three-sphere $S^{3}$ is the union $L=K_{1} \cup \cdots \cup K_{\mu}$ of a finite number of oriented, pairwise disjoint, polygonal simple closed curves, its components. Two such links are ambient isotopic iff there is an orientation-preserving homeomorphism of $S^{3}$ with itself which maps one onto the other in such a way that the indexing and orientations of the components correspond.

Among the invariants of the ambient isotopy type of a link $L$, one of the most important is the group $G=\pi_{1}\left(S^{3}-L\right)$ of $L$. The lower central series $\left\{G_{q}\right\}$ of $G$ is given by $G_{1}=G$ and, for $q \geq 1, G_{q+1}=\left[G, G_{q}\right]$. In this paper we will be concerned with the various Chen groups $G^{\prime \prime} G_{q} / G^{\prime \prime} G_{q+1}$, and also the quotients $G_{q} / G_{q+1}$ of the lower central series; these abelian groups are invariants of $L$ under equivalence relations much coarser than ambient isotopy $[9 ; 14]$, and are of interest only when $\mu>1$.

In the simplest interesting case, $\mu=2, \mathrm{~K}$. Murasugi [11] has shown that if the linking number $l=l\left(K_{1}, K_{2}\right)$ is nonzero, then the Chen groups of $L$ are all determined (up to isomorphism) by $l: G^{\prime \prime} G_{q} / G^{\prime \prime} G_{q+1} \cong \mathbf{Z}^{q-1}$ $\forall q \geq 2$, where $\mathbf{Z}_{l}$ is the cyclic group of order $|l|$. In particular, these three statements are equivalent: $l= \pm 1, G_{2} / G_{3}=0$, and $G^{\prime \prime} G_{q} / G^{\prime \prime} G_{q+1}=0$ $\forall q \geq 2$. (Note that if $G_{2} / G_{3}=0$ then $G_{2}=G_{3}=G_{q} \forall q \geq 2$, and hence $G_{q} / G_{q+1}=0 \forall q \geq 2$.)

Murasugi conjectured that analogously, a link $L$ of $\mu>2$ components with all linking numbers $l\left(K_{i}, K_{j}\right)= \pm 1$ would have $G_{q} / G_{q+1} \cong$ $F_{q} / F_{q+1} \forall q \geq 2$, where $F$ is free on $\mu-1$ generators. This conjecture has been partly verified by S. Kojima [6], and T. Maeda [8] has shown that the hypothesis that all the linking numbers $l\left(K_{i}, K_{j}\right)$ be \pm 1 can be significantly weakened. 
In this paper we completely characterize those links such that $G_{q} / G_{q+1} \cong F_{q} / F_{q+1}$ for all $q \geq 2$. To explain our characterization of such links, we need to introduce the following notation. Let the $\mu \times\left(\begin{array}{c}\mu \\ 2\end{array}\right)$ matrix $\Lambda$, whose rows are indexed by $\{1, \ldots, \mu\}$ and whose columns are indexed by $\{(p, q) \mid 1 \leq p<q \leq \mu\}$, have the entries

$$
\Lambda_{l(p, q)}= \begin{cases}l\left(K_{i}, K_{q}\right), & \text { if } i=p \\ -l\left(K_{l}, K_{p}\right), & \text { if } i=q \\ 0, & \text { if } p \neq i \neq q .\end{cases}
$$

Let $E_{\left(\mu_{2}^{-1}\right)}(\Lambda)$ be the $\left({ }_{2}^{\mu-1}\right)$ th elementary ideal of $\Lambda$, that is, the ideal of $\mathbf{Z}$ generated by the determinants of the various $(\mu-1) \times(\mu-1)$ submatrices of $\Lambda$. (Another description of this ideal is given in §3.) Equivalently, we may use elementary row and column operations to transform $\Lambda$ into a matrix

$$
\left(\begin{array}{ll}
D & 0 \\
0 & 0
\end{array}\right)
$$

where $D$ is a $(\mu-1) \times(\mu-1)$ diagonal matrix, and then $E_{\left(\mu_{2}^{-1}\right)}(\Lambda)$ is the ideal of $\mathbf{Z}$ generated by the product of the diagonal entries of $D$.

TheOREM 1. Let $L$ be a tame link of $\mu \geq 2$ components in $S^{3}, G$ the group of $L$, and $F$ the free group on $\mu-1$ generators. Then any two of these statements are equivalent:

(a) $G_{2} / G_{3} \cong F_{2} / F_{3}$;

(b) $E_{\left({ }^{\mu-1}\right)}(\Lambda)=\mathbf{Z}$;

(c) $G^{\prime \prime} G_{q} / G^{\prime \prime} G_{q+1} \cong F^{\prime \prime} F_{q} / F^{\prime \prime} F_{q+1} \forall q \geq 2$;

(d) $G_{q} / G_{q+1} \cong F_{q} / F_{q+1} \forall q \geq 2$; and

(e) the cup-product pairing

$$
H^{1}\left(S^{3}-L ; \mathbf{Z}\right) \otimes_{\mathbf{Z}} H^{1}\left(S^{3}-L ; \mathbf{Z}\right) \rightarrow H^{2}\left(S^{3}-L ; \mathbf{Z}\right)
$$

is epimorphic.

That (a) implies (b) follows from the theorem of K. -T. Chen [3] that $\Lambda$ is a presentation matrix of the abelian group $G_{2} / G_{3}$, for if $G_{2} / G_{3} \cong$ $F_{2} / F_{3}$ is free abelian of rank $\left(\begin{array}{c}\mu-1 \\ 2\end{array}\right)$, then

$$
E_{\left(\begin{array}{c}
\mu-1 \\
2
\end{array}\right)}\left(G_{2} / G_{3}\right)=E_{\left(\begin{array}{c}
\mu-1 \\
2
\end{array}\right)}(\Lambda)=\mathbf{Z}
$$

(See [12, Chapter 3] for a discussion of the basic properties of the elementary ideals (or "Fitting invariants") of a finitely generated module over a commutative ring with unity.) That (c) is equivalent to (d) has been 
proven by T. Maeda [8]. (Maeda has mentioned to us that he actually announced the equivalence of (a), (c), and (d) at a conference held at the University of Sussex in 1982, but that subsequently he found a gap in the argument he'd devised to deduce (c) from (a).) Clearly (d) implies (a), so the proof of the equivalence of (a), (b), (c), and (d) is completed by verifying the implication (b) $\Rightarrow$ (c). We do this in $\$ 3$, where we also present yet another condition equivalent to those of Theorem 1 . We discuss (e) in $\$ 4$.

Let $\nabla(L) \in \mathbf{Z}\left[t, t^{-1}\right]$ be the Hosokawa polynomial of $L$ [5]. Using the relationship between $\nabla(L)(1)$ and the linking numbers of the components of $L$ with each other, we also prove

Proposition 1. If $\nabla(L)(1)= \pm 1$, then the equivalent conditions of Theorem 1 hold.

2. Some commutative algebra. In this section we present some algebraic notions that will be useful in our proof of the implication (b) $\Rightarrow$ (c). Much of the material involving $I$-adic filtrations, their associated graded modules, and I-adic completions appears in [17, Chapter VIII] and [1, Chapter III] (in a more general context), though our presentation differs somewhat in notation and terminology.

It will be convenient for us to use $P$ to denote a ring that may be either the integers $\mathbf{Z}$ or a prime field (i.e., the rationals $\mathbf{Q}$ or a quotient $\mathbf{Z}_{p}=\mathbf{Z} / p \mathbf{Z}, p$ a prime). For any such $P$, there is a unique homomorphism $\gamma_{P}: \mathbf{Z} \rightarrow P$ with $\gamma_{P}(1)=1$. Also, if $A$ and $B$ are abelian groups with subgroups $C$ and $D$, we use $C \otimes D$ to denote the image of $C \otimes_{\mathrm{z}} D$ in $A \otimes_{\mathrm{z}} B$ (that is, the subgroup of $A \otimes_{\mathrm{z}} B$ generated by the various elements $c \otimes d$ with $c \in C$ and $d \in D$ ).

If $L$ is a link of $\mu$ components in $S^{3}$, and $G$ is the group of $L$, then by Alexander duality the abelianization $H=G / G_{2}$ is the free abelian group on certain generators $t_{1}, \ldots, t_{\mu}$, the meridians of $L$. A group ring $P H$ may be identified, then, with the ring $P\left[t_{1}, \ldots, t_{\mu}, t_{1}^{-1}, \ldots, t_{\mu}^{-1}\right]$ of Laurent polynomials in $t_{1}, \ldots, t_{\mu}$ with coefficients from $P$. The augmentation map $\varepsilon: P H \rightarrow P$ is given by $\varepsilon(h)=1 \forall h \in H$ (or, equivalently, $\varepsilon(f)=f(1, \ldots, 1)$ for a Laurent polynomial $\left.f\left(t_{1}, \ldots, t_{\mu}\right)\right)$; its kernel is the augmentation ideal $\mathrm{IH}$ of $\mathrm{PH}$. Note that $\gamma_{P}$ induces an isomorphism

$$
\gamma_{P^{*}}: P \otimes_{\mathrm{Z}} \mathbf{Z} H \rightarrow P H
$$

under which $P \otimes I H$ is mapped onto $I H$. 
If $B$ is a $P H$-module, it is filtered by the various submodules $(I H)^{s} \cdot B, s \geq 0$. The associated graded module of $B$ is the direct sum

$$
\operatorname{gr}(B)=\bigoplus_{s \geq 0}(I H)^{s} \cdot B /(I H)^{s+1} \cdot B
$$

it is a graded module over the graded ring $\operatorname{gr}(P H)$ in a natural way. We denote by $v$ the order function associated to the filtration $\left\{(I H)^{s} \cdot B\right\}$, that is, for $b \in \bigcap_{s}(I H)^{s} \cdot B, v(b)=\infty$, and for other elements $b$ of $B$, $b \in(I H)^{v(b)} \cdot B-(I H)^{v(b)+1} \cdot B$. For $b \in B$ the initial form in $(b)$ is $\operatorname{in}(b)=b+(I H)^{v(b)+1} \cdot B \in \operatorname{gr}_{v(b)}(B)$, if $v(b)$ is finite, and in $(b)=0$ if $v(b)=\infty$; this defines a function in: $B \rightarrow \operatorname{gr}(B)$.

If $B$ and $C$ are $P H$-modules, and $e: B \rightarrow C$ is a $P H$-epimorphism, it defines a $\operatorname{gr}(P H)$-epimorphism $\operatorname{gr}(e): \operatorname{gr}(B) \rightarrow \operatorname{gr}(C)$ in a natural way. The kernel of $\operatorname{gr}(e)$ is the leading submodule of $\operatorname{ker} e$ in $B$, which we denote in $(\operatorname{ker} e)$; it is the $\operatorname{gr}(P H)$-submodule of $\operatorname{gr}(B)$ consisting of the initial forms of the elements of ker $e$, that is,

$$
\text { in }_{s}(\operatorname{ker} e)=\left((I H)^{s} \cdot B\right) \cap \operatorname{ker} e+(I H)^{s+1} \cdot B /(I H)^{s+1} \cdot B
$$

for $s \geq 0$.

We say an element of $\mathrm{PH}$ is homogeneous of degree $s$ (with respect to $\left.t_{1}-1, \ldots, t_{\mu}-1\right)$ iff it can be expressed as a sum

$$
\sum a_{r_{1}, \ldots, r_{\mu}} \cdot\left(t_{1}-1\right)^{r_{1}} \cdots\left(t_{\mu}-1\right)^{r_{\mu}}
$$

the sum taken over the set of all $\mu$-tuples $\left(r_{1}, \ldots, r_{\mu}\right)$ of non-negative integers with $\Sigma r_{i}=s$, for suitable $a_{r_{1}, \ldots, r_{\mu}} \in P$. (Note that 0 is homogeneous of every degree.) If $Z$ is a free $P H$-module with basis $\left\{z_{i}\right\}$, we say an element of $Z$ is homogeneous of degree $s$ (with respect to this basis) iff it can be expressed as a sum $\sum h_{i} z_{i}$, where each $h_{i}$ is homogeneous of degree $s$, and we denote by $H_{s}(Z)$ the $P$-submodule of $Z$ consisting of the homogeneous elements of degree $s$. Clearly then

Proposition (2.1). For every $s \geq 0$, the initial form function in: $Z \rightarrow \operatorname{gr}(Z)$ defines an isomorphism between $H_{s}(Z)$ and $\operatorname{gr}_{s}(Z)$.

Corollary (2.2). If $Z$ is a free $P H$-module with basis $\left\{z_{1}, \ldots, z_{n}\right\}$, then for $s \geq 0, \operatorname{gr}_{s}(Z)$ is a free P-module of rank $n \cdot\left(\begin{array}{c}\mu+s-1 \\ s\end{array}\right)$.

Proof. It suffices to verify this in case $n=1$.

Clearly $H_{s}(P H)$ is the free $P$-module with basis

$$
\left\{\left(t_{i_{1}}-1\right) \cdots\left(t_{i_{s}}-1\right) \mid 1 \leq i_{1} \leq \cdots \leq i_{s} \leq \mu\right\},
$$

which has $\left(\begin{array}{c}\mu+s-1 \\ s\end{array}\right)$ elements. 
If a submodule of a free $P H$-module $Z$ is generated by homogeneous elements of $Z$, then its leading submodule in $Z$ is simple to describe, as we see in the next two propositions.

LEMMA (2.3). Let $Y$ and $Z$ be free $P H$-modules with bases $\left\{y_{i}\right\}$ and $\left\{z_{j}\right\}$, respectively, and let $f: Y \rightarrow Z$ be a PH-homomorphism with the property that for some fixed $r \geq 0, f\left(y_{i}\right) \in H_{r}(Z) \forall i$. Then $\operatorname{in}_{s}(f(Y))=0$ for $s<r$, and $\operatorname{in}_{s}(f(Y))=f\left(H_{s-r}(Y)\right)+(I H)^{s+1} \cdot Z /(I H)^{s+1} \cdot Z$ for $s \geq r$.

Proof. Clearly in $(f(Y))=0$ for $s<r$.

Suppose $s \geq r$ and $y=\sum r_{i} y_{i} \in Y$ has $f(y) \in(I H)^{s} \cdot Z$. If $r_{i} \in$ $(I H)^{s-r} \forall i$, then $f(y)+(I H)^{s+1} \cdot Z=f(h)+(I H)^{s+1} \cdot Z$, where $h$ is the homogeneous element of $Y$ whose initial form is in $(y)$.

On the other hand, suppose some $r_{i}$ is not in $(I H)^{s-r}$. Let $s-r-t$ $=\min \left\{v\left(r_{i}\right)\right\}$. We proceed by induction on $t$, having just verified the case $t=0$. For each $i$, let $h_{i}$ be 0 (if $v\left(r_{i}\right)>s-r-t$ ), or the homogeneous element of $P H$ whose initial form is $r_{i}+(I H)^{s-r-t+1}$ (if $v\left(r_{i}\right)=s-r-$ $t)$. Then $f\left(\sum h_{i} y_{i}\right)$ is a homogeneous element of $Z$ of degree $s-t$, clearly. In addition, $r_{i}-h_{i} \in(I H)^{s-r-t+1} \forall i$. Since $f(y) \in(I H)^{s-t+1} \cdot Z$, it follows that $f\left(\sum h_{i} y_{i}\right) \in(I H)^{s-t+1} \cdot Z$, and hence $f\left(\sum h_{i} y_{i}\right)=0$, by Proposition (2.1). Thus $f(y)=f\left(\sum\left(r_{i}-h_{i}\right) y_{i}\right)$, and since $r_{i}-h_{i} \in$ $(I H)^{s-r-t+1} \forall i$, we may apply the inductive hypothesis to conclude that $f(y)+(I H)^{s+1} \cdot Z=f(h)+(I H)^{s+1} \cdot Z$ for some $h \in H_{s-r}(Y)$.

Generalizing Lemma (2.3) inductively, we have

Proposition (2.4). Let $Y$ and $Z$ be free PH-modules with bases $\left\{y_{i}\right\}$ and $\left\{z_{j}\right\}$, respectively, and let $f: Y \rightarrow Z$ be a PH-homomorphism with the property that $f\left(y_{i}\right)$ is homogeneous $\forall i$. For $k \geq 0$, let $\left\{y_{i k}\right\}$ be the set of those $y_{i}$ with $v\left(f\left(y_{i}\right)\right)=k$, and $Y_{k}$ the submodule of $Y$ freely generated by $\left\{y_{i k}\right\}$ (if there are no $y_{i k}, Y_{k}=0$ ). Then

$$
\text { in }_{s}(f(Y))=\sum_{k=0}^{s} f\left(H_{s-k}\left(Y_{k}\right)\right)+(I H)^{s+1} \cdot Z /(I H)^{s+1} \cdot Z
$$

for every $s \geq 0$.

Proof. Suppose $s \geq 0$, and let $J$ be the right-hand side of the asserted equality. Obviously in ${ }_{s}(f(Y)) \supseteq J$, so it suffices to verify the opposite inclusion. 
Every element of $\operatorname{in}_{s}(f(Y))$ is $f(x)+(I H)^{s+1} \cdot Z$ for some $x \in$ $\sum_{k=0}^{s} Y_{k}$, say $x=\sum_{k=0}^{s} x_{k}$. If $v\left(f\left(x_{k}\right)\right)=s \forall k$, then $f(x)+(I H)^{s+1} \cdot Z$ $=\sum \operatorname{in}\left(f\left(x_{k}\right)\right)$, and we may apply Lemma (2.3) to find, for each $k \in$ $\{0, \ldots, s\}$, an $h_{k} \in H_{s-k}\left(Y_{k}\right)$ with $\operatorname{in}\left(f\left(x_{k}\right)\right)=\operatorname{in}\left(f\left(h_{k}\right)\right)$. Then $f(x)+$ $(I H)^{s+1} \cdot Z=\sum \operatorname{in}\left(f\left(h_{k}\right)\right) \in J$.

On the other hand, suppose $v\left(f\left(x_{k}\right)\right)<s$ for some $k \in\{0, \ldots, s\}$, and let $s-t=\min \left\{v\left(f\left(x_{k}\right)\right)\right\}$. We proceed by induction on $t$, having just dealt with $t=0$. For $k \in\{0, \ldots, s\}$ let $h_{k}=0$ if $v\left(f\left(x_{k}\right)\right)>s-t$, and otherwise let $h_{k} \in H_{s-t-k}\left(Y_{k}\right)$ have in $\left(f\left(h_{k}\right)\right)=\operatorname{in}\left(f\left(x_{k}\right)\right)$. (Such an $h_{k}$ exists by Lemma (2.3).) Since $v(f(x))=s>s-t$, it must be that $\sum \operatorname{in}\left(f\left(h_{k}\right)\right)=0$, and hence $\sum f\left(h_{k}\right)=0$ by Proposition (2.1). Thus $f(x)$ $+(I H)^{s+1} \cdot Z=f\left(\sum_{k=0}^{s}\left(x_{k}-h_{k}\right)\right)+(I H)^{s+1} \cdot Z$. Since $v\left(f\left(x_{k}-h_{k}\right)\right)$ $>s-t$ for every $k$, we may apply the inductive hypothesis to conclude that $f\left(\Sigma\left(x_{k}-h_{k}\right)\right)+(I H)^{s+1} \cdot Z \in J$.

We will call a map $f$ that satisfies the hypothesis of Proposition (2.4) homogeneous; a $\mathrm{PH}$-module possesses a homogeneous presentation (or is homogeneously presentable) iff it is isomorphic to the cokernel of some homogeneous map.

The augmentation ideal $I H$ is an example of a homogeneously presentable $\mathbf{Z} H$-module. Recall [7, p. 189] that there is an exact sequence

$$
Z_{4} \stackrel{\zeta_{4}}{\rightarrow} Z_{3} \stackrel{\zeta_{3}}{\rightarrow} Z_{2} \stackrel{\zeta_{2}}{\rightarrow} Z_{1} \stackrel{\zeta_{1}}{\rightarrow} I H \rightarrow 0
$$

of $\mathbf{Z} H$-modules, where $Z_{4}$ is free on $\left\{z_{\imath \jmath k l} \mid 1 \leq i<j<k<l \leq \mu\right\}, Z_{3}$ is free on $\left\{z_{\imath \jmath k} \mid 1 \leq i<j<k \leq \mu\right\}, Z_{2}$ is free on $\left\{z_{i j} \mid 1 \leq i<j \leq \mu\right\}, Z_{1}$ is free on $\left\{z_{l} \mid 1 \leq i \leq \mu\right\}$,

$$
\begin{gathered}
\zeta_{1}\left(z_{i}\right)=t_{i}-1, \zeta_{2}\left(z_{i j}\right)=\left(t_{l}-1\right) z_{J}-\left(t_{J}-1\right) z_{l}, \\
\zeta_{3}\left(z_{i j k}\right)=\left(t_{i}-1\right) z_{j k}-\left(t_{J}-1\right) z_{i k}+\left(t_{k}-1\right) z_{i J},
\end{gathered}
$$

and

$$
\begin{aligned}
\zeta_{4}\left(z_{i j k l}\right)= & \left(t_{l}-1\right) z_{j k l}-\left(t_{j}-1\right) z_{i k l} \\
& +\left(t_{k}-1\right) z_{i j l}-\left(t_{l}-1\right) z_{i j k} .
\end{aligned}
$$

Proposition (2.5). For $s>0, \operatorname{in}_{s}\left(\zeta_{3}\left(Z_{3}\right)\right)$ is a direct summand of $\mathrm{gr}_{s}\left(Z_{2}\right)$ whose rank is

$$
\left(\begin{array}{c}
s+1 \\
2
\end{array}\right) \cdot\left(\begin{array}{c}
\mu+s-1 \\
s+2
\end{array}\right)
$$


Proof. If $s=1$,

$$
\operatorname{in}_{s}\left(\zeta_{3}\left(Z_{3}\right)\right)=\zeta_{3}\left(H_{0}\left(Z_{3}\right)\right)+(I H)^{2} \cdot Z_{2} /(I H)^{2} \cdot Z_{2}
$$

is generated by the $\left(\begin{array}{c}\mu \\ 3\end{array}\right)$ elements $\zeta_{3}\left(z_{i j k}\right)+(I H)^{2} \cdot Z_{2}$. To verify that these elements are linearly independent over $\mathbf{Z}$, and that $\operatorname{in}_{s}\left(\zeta_{3}\left(Z_{3}\right)\right)$ is a direct summand of $\operatorname{gr}_{s}\left(Z_{2}\right)$, note that $\operatorname{gr}_{s}\left(Z_{2}\right)$ is a free abelian group with a basis consisting of the various elements $\left(t_{i}-1\right) z_{j k}+(I H)^{2} \cdot Z_{2}$, and we can obtain a new basis by replacing $\left(t_{i}-1\right) z_{j k}+(I H)^{2} \cdot Z_{2}$ by $\zeta_{3}\left(z_{i j k}\right)+(I H)^{2} \cdot Z_{2}$ whenever $i<j$.

If $s \geq 2$,

$$
\operatorname{in}_{s}\left(\zeta_{3}\left(Z_{3}\right)\right)=\zeta_{3}\left(H_{s-1}\left(Z_{3}\right)\right)+(I H)^{s+1} \cdot Z_{2} /(I H)^{s+1} \cdot Z_{2}
$$

is generated by the various elements

$$
\begin{aligned}
\zeta_{3}\left(\left(t_{r_{1}}-1\right) \cdots\left(t_{r_{s-1}}-1\right) z_{i j k}\right)+(I H)^{s+1} \cdot & Z_{2}, \\
& 1 \leq r_{1} \leq \cdots \leq r_{s-1} \leq \mu .
\end{aligned}
$$

Since $\zeta_{3} \zeta_{4}=0$, those

$$
\zeta_{3}\left(\left(t_{r_{1}}-1\right) \cdots\left(t_{r_{s-1}}-1\right) z_{i j k}\right)+(I H)^{s+1} \cdot Z_{2}
$$

with $r_{1} \geq i$ suffice to generate in ${ }_{s}\left(\zeta_{3}\left(Z_{3}\right)\right)$. For a given $i \in\{1, \ldots, \mu-2\}$, the number of pairs $(j, k)$ with $1 \leq i<j<k \leq \mu$ is $\left(\begin{array}{c}\mu-i \\ 2\end{array}\right)$, and the number of $(s-1)$-tuples $\left(r_{1}, \ldots, r_{s-1}\right)$ with $i \leq r_{1} \leq \cdots \leq r_{s-1} \leq \mu$ is $\left(\begin{array}{c}\mu+s-i-1 \\ s-1\end{array}\right)$. Thus the number of elements of this generating set of in ${ }_{s}\left(\zeta_{3}\left(Z_{3}\right)\right)$ is

$$
\sum_{i=1}^{\mu-2}\left(\begin{array}{c}
\mu-i \\
2
\end{array}\right)\left(\begin{array}{c}
\mu+s-i-1 \\
s-1
\end{array}\right)=\left(\begin{array}{c}
s+1 \\
2
\end{array}\right) \cdot \sum_{i=1}^{\mu-2}\left(\begin{array}{c}
\mu+s-i-1 \\
s+1
\end{array}\right),
$$

and the latter sum is easily seen to be $\left(\begin{array}{c}\mu+s-1 \\ s+2\end{array}\right)$. To verify that this generating set is linearly independent over $\mathbf{Z}$, and that in ${ }_{s}\left(\zeta_{3}\left(Z_{3}\right)\right)$ is a direct summand of $\operatorname{gr}_{s}\left(Z_{2}\right)$, note that $\operatorname{gr}_{s}\left(Z_{2}\right)$ is a free abelian group with a basis consisting of the various elements

$$
\left(t_{i}-1\right)\left(t_{r_{1}}-1\right) \cdots\left(t_{r_{s-1}}-1\right) z_{j k}+(I H)^{s+1} \cdot Z_{2}
$$

with $i \leq r_{1} \leq \cdots \leq r_{s-1}$, and we can obtain a new basis by replacing

$$
\left(t_{i}-1\right)\left(t_{r_{1}}-1\right) \cdots\left(t_{r_{s-1}}-1\right) z_{j k}+(I H)^{s+1} \cdot Z_{2}
$$

by

$$
\zeta_{3}\left(\left(t_{r_{1}}-1\right) \cdots\left(t_{r_{s-1}}-1\right) z_{i j k}\right)+(I H)^{s+1} \cdot Z_{2}
$$

whenever $i<j$. 
If $B$ is a $\mathrm{Z} H$-module then for any $P, P \otimes_{\mathrm{Z}} B$ is a $P H$-module under the action $\gamma_{p^{*}}(x \otimes h) \cdot(y \otimes b)=(x y) \otimes(h b)$. For every $s \geq 0, \quad \gamma_{P^{*}}\left(P \otimes(I H)^{s}\right)=(I H)^{s}$, and so certainly $(I H)^{s} \cdot\left(P \otimes_{\mathrm{Z}} B\right)=$ $P \otimes(I H)^{s} \cdot B$. A natural homomorphism $\delta_{P}: P \otimes_{\mathrm{z}} \operatorname{gr}(B) \rightarrow \operatorname{gr}\left(P \otimes_{\mathrm{z}} B\right)$ may be defined by

$$
\delta_{P}\left(x \otimes\left(b+(I H)^{s+1} \cdot B\right)\right)=x \otimes b+(I H)^{s+1} \cdot\left(P \otimes_{\mathbf{z}} B\right)
$$

for $x \in P$ and $b \in(I H)^{s} \cdot B$.

Proposition (2.6). For any $\mathbf{Z} H$-module $B, \delta_{P}$ is an isomorphism.

Proof. Suppose $s \geq 0$, and let $c:(I H)^{s} \cdot B \rightarrow \mathrm{gr}_{s}(B)$ be the canonical map onto the quotient. Then

$$
\mathrm{id}_{P} \otimes c: P \otimes_{\mathrm{Z}}(I H)^{s} \cdot B \rightarrow P \otimes_{\mathrm{Z}} \operatorname{gr}_{s}(B)
$$

is an epimorphism whose kernel is $P \otimes(I H)^{s+1} \cdot B$, by the right exactness of tensor products. Let $i:(I H)^{s} \cdot B \rightarrow B$ be the inclusion map, and $c_{P}:(I H)^{s} \cdot\left(P \otimes_{\mathrm{z}} B\right) \rightarrow \mathrm{gr}_{s}\left(P \otimes_{\mathrm{z}} B\right)$ the canonical map onto the quotient. Since

$$
\begin{gathered}
\left(\mathrm{id}_{P} \otimes i\right)\left(P \otimes_{\mathrm{Z}}(I H)^{s} \cdot B\right)=(I H)^{s} \cdot\left(P \otimes_{\mathrm{Z}} B\right) \\
c_{P}\left(\mathrm{id}_{P} \otimes i\right): P \otimes_{\mathrm{Z}}(I H)^{s} \cdot B \rightarrow \mathrm{gr}_{s}\left(P \otimes_{\mathrm{Z}} B\right)
\end{gathered}
$$

is an epimorphism; since

$$
\left(\mathrm{id}_{P} \otimes i\right)\left(P \otimes(I H)^{s+1} \cdot B\right)=(I H)^{s+1} \cdot\left(P \otimes_{\mathrm{Z}} B\right)=\operatorname{ker} c_{P},
$$

this epimorphism induces an isomorphism

$$
\delta_{P}: P \otimes_{\mathrm{z}} \operatorname{gr}_{s}(B) \rightarrow \operatorname{gr}_{s}\left(P \otimes_{\mathrm{z}} B\right) .
$$

If $B$ is a $P H$-module, the order function $v$ defines a metric on $B / \cap_{s}(I H)^{s} \cdot B$ by

$$
d(\bar{a}, \bar{b})=\exp (-v(a-b))
$$

where $\bar{a}$ and $\bar{b}$ are the elements of $B / \bigcap_{s}(I H)^{s} \cdot B$ determined by $a$ and $b$. The completion $\hat{B}$ of this metric space is the $I H$-adic completion of $B$; it is a $\widehat{P H}$-module in a natural way. We denote the natural mapping $B \rightarrow \hat{B}$ by $h$; in case $h$ is injective (i.e., if $\bigcap_{s}(I H)^{s} \cdot B=0$ ) we may suppress it, and regard $\mathrm{B}$ as a subset of $\hat{B}$. If $B$ is finitely generated, there is an isomorphism $\widehat{P H} \otimes_{P H} B \rightarrow \hat{B}$ under which the image of $x \otimes b$ is $x \cdot h(b)$ [1, III, §3.4]; consequently, if $B$ is the free $P H$-module on a finite set of generators, $\hat{B}$ is the free $\widehat{P H}$-module on the same set. 
Just as $P H$ may be identified with the ring $P\left[t_{1}, \ldots, t_{\mu}, t_{1}^{-1}, \ldots, t_{\mu}^{-1}\right]$ of Laurent polynomials, its $I H$-adic completion may also be identified with a familiar ring: the imbedding of $P H$ in the formal power series ring $P\left[\left[u_{1}, \ldots, u_{\mu}\right]\right]$ given by $t_{i} \mapsto 1-u_{i}$ extends to an isomorphism between $\widehat{P H}$ and $P\left[\left[u_{1}, \ldots, u_{\mu}\right]\right]$ (see $[9, \S 3]$ for the case $P=\mathbf{Z}$; the general argument is similar).

Let $\overparen{I H}$ be the ideal of $\widehat{P H}$ generated by $I H$. Then every $\widehat{P H}$-module possesses an $\widehat{I H}$-adic filtration defined in the obvious way, and this filtration produces an associated graded module as before. If $B$ is a finitely generated $P H$-module the natural mapping $h: B \rightarrow \hat{B}$ defines an isomorphism $\operatorname{gr}(h): \operatorname{gr}(B) \rightarrow \operatorname{gr}(\hat{B})$ of graded modules over the graded ring $\operatorname{gr}(P H) \cong \operatorname{gr}(\widehat{P H})$.

We close this section with two properties of elementary ideals. Let $R$ be a commutative ring with unity, $X$ and $Y$ free $R$-modules of finite ranks $m$ and $n$, respectively, with $m \leq n$. Let $f: X \rightarrow Y$ be an $R$-homomorphism, $M$ an $m \times n$ matrix that represents $f$ with respect to some bases of $X$ and $Y$, and $E_{n-m}(M)$ the ideal of $R$ generated by the determinants of the various $m \times m$ submatrices of $M$.

LEMMA (2.7). If $E_{n-m}(M)$ is not annihilated by any nonzero element of $R, f$ is a monomorphism. Furthermore, $f$ is a split monomorphism iff $E_{n-m}(M)=R$.

Proof. Let $M=\left(m_{i j}\right)$ be the matrix of $f$ with respect to the bases $\left\{x_{1}, \ldots, x_{m}\right\}$ of $X$ and $\left\{y_{1}, \ldots, y_{n}\right\}$ of $Y$.

Suppose, first, that ann $E_{n-m}(M)=0$, and $x=\sum a_{i} x_{i} \in \operatorname{ker} f$. Then for every $j \in\{1, \ldots, n\}, \sum a_{i} m_{i j}=0$; that is, the $a_{i}$ are the coefficients in a relation between the rows of $M$. It follows that $a_{i} \operatorname{det} N=0$ for every $i$ and every $m \times m$ submatrix $N$ of $M$, so $a_{1}, \ldots, a_{m} \in \operatorname{ann} E_{n-m}(M)$; thus $x=0$.

Suppose $E_{n-m}(M)=R$. Then $f$ is a monomorphism, and furthermore, coker $f$ is a projective $R$-module [12, p. 122], so $f$ is split. Conversely, suppose $f$ is a split monomorphism, and let $Y=f(X) \oplus C$. Then $X$ and $f(X)$ are isomorphic $R$-modules, so they have the same elementary ideals, or "Fitting invariants". (See [12, Chapter 3] for an account of the basic properties of these invariants.) It follows that $R=E_{n}(Y)=$ $E_{n-m}(C)$, and since $M$ is a presentation matrix for $C, E_{n-m}(C)=$ $E_{n-m}(M)$.

3. The completion of the Alexander invariant. The Alexander invariant $B_{L}$ of a link $L$ is the abelianization $G^{\prime} / G^{\prime \prime}=B_{L}$ of the commutator subgroup of its group, considered as an $H$-module with the action given 
by $\left(g G^{\prime}\right) \cdot\left(c G^{\prime \prime}\right)=g c g^{-1} G^{\prime \prime}$ for $g \in G$ and $c \in G^{\prime}$. This module has the property that

$$
G^{\prime \prime} G_{q} / G^{\prime \prime} G_{q+1}=(I H)^{q-2} \cdot B_{L} /(I H)^{q-1} \cdot B_{L}=\operatorname{gr}_{q-2}\left(B_{L}\right),
$$

and hence $G^{\prime \prime} G_{q} / G^{\prime \prime} G_{q+1} \cong \operatorname{gr}_{q-2}\left(\hat{B}_{L}\right)$, for $q \geq 2[9]$.

Let $Y=Y_{0}$ be the free $\mathbf{Z} H$-module on the set $\left\{x_{1}, \ldots, x_{\mu}\right\}$, and let $\lambda_{0}: Y_{0} \rightarrow Z_{2}$ be the $\mathbf{Z} H$-homomorphism whose matrix (with respect to the bases $\left\{x_{l}\right\}$ and $\left.\left\{z_{i j}\right\}\right)$ is the matrix $\Lambda$ defined in the introduction. For $1 \leq r \leq \mu$, let $Y_{r}$ be the submodule of $Y_{0}$ generated by those $x_{i}$ other than $x_{r}$, and $\lambda_{r}$ the restriction of $\lambda_{0}$ to $Y_{r}$. For each $r$, let $\hat{\lambda}_{r}: \hat{Y}_{r} \rightarrow \hat{Z}_{2}$ be the unique continuous extension of $\lambda_{r}$.

It was proven in [16] that there is a $\widehat{\mathbf{Z H}}$-homomorphism $\nu_{0}: \hat{Y}_{0} \rightarrow \hat{Z}_{2}$ such that for every $r \in\{0, \ldots, \mu\}$ there is an exact sequence

$$
\hat{Y}_{r} \oplus \hat{Z}_{3} \stackrel{\left\langle\nu_{r}, \hat{\xi}_{3}\right\rangle}{\rightarrow} \hat{Z}_{2} \stackrel{\tau}{\rightarrow} \hat{B}_{L} \rightarrow 0
$$

where $\nu_{r}$ is the restriction of $\nu_{0}$ to $\hat{Y}_{r}$. It will not be necessary for us to recall the definition of this map $\nu_{0}$, but two properties will be useful: $\nu_{0}\left(x_{i}\right) \equiv \hat{\lambda}_{0}\left(x_{i}\right)$ (modulo $\left.\widehat{I H} \cdot \hat{Z}_{2}\right) \forall i$; and if $L$ happens to be the unlink of $\mu$ components, then $\nu_{0}=0$.

The second property leads directly to a well-known formula.

Proposition (3.2). Let $\Phi$ be the free group on $\mu$ generators. Then for $q \geq 2, \Phi^{\prime \prime} \Phi_{q} / \Phi^{\prime \prime} \Phi_{q+1}$ is a free abelian group of rank $(q-1) \cdot(\underset{q}{\mu+q-2})$.

Proof. Let $L_{0}$ be the unlink of $\mu$ components; its group is $\Phi$. In the exact sequence (3.1), $\nu_{0}=0$, so $\tau$ is a $\widehat{\mathbf{Z H}}$-epimorphism whose kernel is $\hat{\zeta}_{3}\left(\hat{Z}_{3}\right)$. For every $s \geq 0$, then, $\operatorname{gr}_{s}(\tau)$ is an epimorphism of $\operatorname{gr}_{s}\left(\hat{Z}_{2}\right)=$ $\operatorname{gr}_{s}\left(Z_{2}\right)$ onto $\operatorname{gr}_{s}\left(\hat{B}_{L_{0}}\right) \cong \Phi^{\prime \prime} \Phi_{s+2} / \Phi^{\prime \prime} \Phi_{s+3}$ whose kernel is $\operatorname{in}_{s}\left(\hat{\zeta}_{3}\left(\hat{Z}_{3}\right)\right)=$ in $_{s}\left(\zeta_{3}\left(Z_{3}\right)\right)$. By Corollary (2.2) and Proposition (2.5), it follows that $\Phi^{\prime \prime} \Phi_{s+2} / \Phi^{\prime \prime} \Phi_{s+3}$ is free abelian of rank

$$
\begin{aligned}
\left(\begin{array}{l}
\mu \\
2
\end{array}\right) & \cdot\left(\begin{array}{c}
\mu+s-1 \\
s
\end{array}\right)-\left(\begin{array}{c}
s+1 \\
2
\end{array}\right) \cdot\left(\begin{array}{c}
\mu+s-1 \\
s+2
\end{array}\right) \\
& =\left(\begin{array}{c}
\mu+s-1 \\
s+2
\end{array}\right) \cdot\left(\frac{(s+1)(s+2)}{(\mu-2)(\mu-1)}\left(\begin{array}{c}
\mu \\
2
\end{array}\right)-\left(\begin{array}{c}
s+1 \\
2
\end{array}\right)\right) \\
& =\left(\begin{array}{c}
\mu+s-1 \\
s+2
\end{array}\right) \cdot\left(\frac{s+1}{\mu-2}\right) \cdot\left(\frac{(s+2) \mu}{2}-\frac{s(\mu-2)}{2}\right) \\
& =\left(\frac{s+1}{\mu-2}\right) \cdot\left(\begin{array}{c}
\mu+s-1 \\
s+2
\end{array}\right) \cdot(\mu+s) \\
& =(s+1) \cdot\left(\begin{array}{c}
\mu+s \\
s+2
\end{array}\right) .
\end{aligned}
$$


We conclude that if $F$ is the free group on $\mu-1$ generators, then for $q \geq 2, F^{\prime \prime} F_{q} / F^{\prime \prime} F_{q+1}$ is a free abelian group of $\operatorname{rank}(q-1) \cdot\left(\begin{array}{c}\mu+q-3 \\ q\end{array}\right)$.

For $r \in\{0, \ldots, \mu\}$, let $\left\langle\lambda_{r}, \zeta_{3}\right\rangle: Y_{r} \oplus Z_{3} \rightarrow Z_{2}$ be the obvious map, and let $B_{\Lambda}=\operatorname{coker}\left\langle\lambda_{r}, \zeta_{3}\right\rangle$. (Note that since $\Sigma \lambda_{0}\left(x_{i}\right)=0$, the image of $\left\langle\lambda_{r}, \zeta_{3}\right\rangle$ is independent of $r$; we will generally take $r=\mu$ in this situation, if only for definiteness' sake.) Our strategy in verifying the implication (b) $\Rightarrow$ (c) is to describe $\operatorname{gr}\left(B_{\Lambda}\right)$ when (b) holds, and then to show that (b) implies that $\operatorname{gr}\left(B_{\Lambda}\right)$ and $\operatorname{gr}\left(B_{L}\right)$ are isomorphic.

It is convenient to give a more explicit description of the ideal $E_{\left(\begin{array}{l}\mu-1 \\ 2\end{array}\right)}(\Lambda)$.

Proposition (3.3). The ideal $E_{\left(\begin{array}{c}\mu-1 \\ \text { ( }\end{array}\right)}(\Lambda)$ of $\mathbf{Z}$ is generated by the collection of all products

$$
\prod_{k=1}^{\mu-1} l\left(K_{i_{k}}, K_{j_{k}}\right)
$$

such that $j_{1}, \ldots, j_{\mu-1}$ are pairwise distinct and $i_{k} \notin\left\{j_{k}, \ldots, j_{\mu-1}\right\} \forall k$.

Proof. Let $E$ be the ideal generated by these products.

Suppose $M$ is a $(\mu-1) \times(\mu-1)$ submatrix of $\Lambda$ with det $M \neq 0$. Note that each column of $\Lambda$ either has no nonzero entries, or else has two nonzero entries which are negatives of each other. $M$ must have some column with precisely one nonzero entry; that is, there must be $i_{1} \neq j_{1} \in$ $\{1, \ldots, \mu\}$ such that $M$ involves the $\left(i_{1}, j_{1}\right)$ or $\left(j_{1}, i_{1}\right)$ column of $\Lambda$, and the $j_{1}$ th row, but not the $i_{1}$ th row. The submatrix of $M$ obtained by deleting this row and column must also have nonzero determinant, so it too must have a column with precisely one nonzero entry; that is, there must be $i_{2} \neq j_{2} \in\{1, \ldots, \mu\}$ such that this submatrix involves the $\left(i_{2}, j_{2}\right)$ or $\left(j_{2}, i_{2}\right)$ column of $\Lambda$, and the $j_{2}$ th row, but not the $i_{2}$ th row. Note that $j_{2}$ cannot be $i_{1}$ or $j_{1}$, since this submatrix involves neither the $i_{1}$ th nor the $j_{1}$ th row of $\Lambda$. Continuing in this vein, we conclude that there are $i_{1}, \ldots, i_{\mu-1}, j_{1}, \ldots, j_{\mu-1} \in\{1, \ldots, \mu\}$ such that $j_{1}, \ldots, j_{\mu-1}$ are pairwise distinct, $i_{k} \notin\left\{j_{k}, \ldots, j_{\mu-1}\right\} \forall k, M$ involves the $j_{k}$ th row of $\Lambda \forall k$, and $M$ involves the $\left(i_{k}, j_{k}\right)$ or $\left(j_{k}, i_{k}\right)$ column of $\Lambda \forall k$. It follows that

$$
\operatorname{det} M= \pm \prod_{k=1}^{\mu-1} l\left(K_{i_{k}}, K_{j_{k}}\right) \in E .
$$

Thus $E_{\left(\frac{\mu-1}{2}\right)}(\Lambda) \subseteq E$. 
To verify that $E_{\left(\mu_{2}^{-1}\right)}(\Lambda) \supseteq E$, note that if $i_{1}, \ldots, i_{\mu-1}, j_{1}, \ldots, j_{\mu-1}$ are as described then the $(\mu-1) \times(\mu-1)$ submatrix of $\Lambda$ which involves the $j_{k}$ th row and $\left(i_{k}, j_{k}\right)$ or $\left(j_{k}, i_{k}\right)$ column $\forall k$ has determinant equal to

$$
\pm \prod_{k=1}^{\mu-1} l\left(K_{i_{k}}, K_{j_{k}}\right)
$$

Let $\Gamma$ be the graph with vertices $\gamma_{1}, \ldots, \gamma_{\mu}$, which has an edge $\left\{\gamma_{i}, \gamma_{j}\right\}$ whenever $i \neq j$ and $l\left(K_{l}, K_{j}\right) \neq 0$. Then we may rephrase Proposition (3.3) in the following way: $E_{\left(\mu_{2}-1\right)}(\Lambda) \neq 0$ iff $\Gamma$ is connected, and if so then $E_{\left(\mu_{2}^{-1}\right)}(\Lambda)$ is the ideal of $\mathbf{Z}$ generated by the set of all products

$$
\prod_{k=1}^{\mu-1} l\left(K_{i_{k}}, K_{j_{k}}\right)
$$

such that $\left\{\gamma_{i_{1}}, \gamma_{j_{1}}\right\}, \ldots,\left\{\gamma_{i_{\mu-1}}, \gamma_{j_{\mu-1}}\right\}$ are the edges of a spanning tree in $\Gamma$.

Corollary (3.4). If $E_{\left(\mu_{2}^{-1}\right)}(\Lambda)=\mathbf{Z}$, then id $\otimes\left(\zeta_{2} \lambda_{\mu}\right): P \otimes_{\mathrm{Z}} Y_{\mu} \rightarrow$ $P \otimes_{\mathrm{z}} Z_{1}$ is injective for every $P$.

Proof. Note that $\sum \lambda_{0}\left(x_{i}\right)=0$, and so if $1 \leq r \leq \mu$ then

$$
\left(\mathrm{id} \otimes\left(\zeta_{2} \lambda_{\mu}\right)\right)\left(\sum_{i<\mu} w_{l} \otimes a_{\imath} x_{\imath}\right)=0
$$

iff

$$
\left(\mathrm{id} \otimes\left(\zeta_{2} \lambda_{r}\right)\right)\left(\sum_{r \neq i<\mu} w_{i} \otimes a_{i} x_{i}-\sum_{i \neq r} w_{r} \otimes a_{r} x_{i}\right)=0 .
$$

It suffices, then, to show that id $\otimes\left(\zeta_{2} \lambda_{r}\right)$ is injective for some $r$. Also, note that for each $i$,

$$
\zeta_{2} \lambda_{0}\left(x_{i}\right)=\sum_{j \neq i} l\left(K_{i}, K_{j}\right) \cdot\left(\left(t_{i}-1\right) z_{j}-\left(t_{j}-1\right) z_{i}\right) .
$$

By the proposition, there must be $i_{1}, \ldots, i_{\mu-1}, j_{1}, \ldots, j_{\mu-1}$ with $j_{1}, \ldots, j_{\mu-1}$ pairwise distinct, $i_{k} \notin\left\{j_{k}, \ldots, j_{\mu-1}\right\} \forall k$, and $l\left(K_{i_{k}}, K_{J_{k}}\right) \not \equiv 0$ (modulo char $P$ ) $\forall k$. We claim that id $\otimes\left(\zeta_{2} \lambda_{i_{1}}\right)$ is injective; by Lemma (2.7), it suffices to show that if $M$ is the matrix representing $\zeta_{2} \lambda_{0}$ with respect to the bases $\left\{x_{i}\right\}$ and $\left\{z_{i}\right\}$, then the submatrix $N$ obtained from $M$ by deleting its $i_{1}$ th row and column has $\gamma_{P^{*}}(1 \otimes \operatorname{det} N) \neq 0$; or equivalently, that if $p=\operatorname{char} P$ then $\operatorname{det} N \not \equiv 0($ modulo $p)$.

For $2 \leq m \leq \mu$, let $N_{m}$ be the submatrix of $M$ obtained by deleting its $i_{1}$ th row and column, and its $j_{k}$ th row and column for $k \geq m$. (In 
particular, $N_{\mu}=N$.) Note that $\operatorname{det} N_{2} \equiv l\left(K_{i_{1}}, K_{j_{1}}\right) \cdot\left(1-t_{i_{1}}\right) \not \equiv 0$ (modulo $\left.\left(p, t_{j_{1}}-1, \ldots, t_{j_{\mu-1}}-1\right)\right)$. Proceeding inductively, suppose $m \geq 2$ and $\operatorname{det} N_{m} \not \equiv 0$ (modulo $\left(p, t_{j_{m-1}}-1, \ldots, t_{j_{\mu-1}}-1\right)$ ). Note that

$$
\operatorname{det} N_{m+1} \equiv\left(\sum_{j \neq j_{m}} l\left(K_{j}, K_{j_{m}}\right)\left(1-t_{j}\right)\right) \cdot \operatorname{det} N_{m}\left(\operatorname{modulo}\left(t_{j_{m}}-1\right)\right),
$$

and since $i_{m} \notin\left\{j_{m}, \ldots, j_{\mu-1}\right\}$, the former factor is not congruent to zero modulo $\left(p, t_{j_{m}}-1, \ldots, t_{j_{\mu-1}}-1\right)$, so

$$
\operatorname{det} N_{m+1} \not \equiv 0\left(\operatorname{modulo}\left(p, t_{j_{m}}-1, \ldots, t_{j_{\mu-1}}-1\right)\right) \text {. }
$$

We conclude that $N=N_{\mu}$ has $\operatorname{det} N \not \equiv 0\left(\right.$ modulo $\left(p, t_{j_{\mu-1}}-1\right)$ ), so certainly det $N \not \equiv 0$ (modulo $p$ ).

Alternatively, an inductive argument using Torres' second relation [15] can be used to show that the Alexander polynomial $\Delta$ of $L$ has $v\left(\gamma_{P^{*}}(1 \otimes \Delta)\right)=\mu-2$. Since

$$
\Delta \cdot I H+(I H)^{\mu}=E_{1}(M)+(I H)^{\mu},
$$

and this equation remains true if the $i_{1}$ th row of $M$ is deleted [16, Corollary (4.3), with $q=2$ ], the matrix representing $\zeta_{2} \lambda_{i_{1}}$ must have some $(\mu-1) \times(\mu-1)$ submatrix $N^{\prime}$ with $v\left(\gamma_{P^{*}}\left(1 \otimes \operatorname{det} N^{\prime}\right)\right)=\mu-1<\infty$, and so by Lemma (2.7), id $\otimes\left(\zeta_{2} \lambda_{i_{1}}\right)$ must be injective.

It is worth noting that for a particular $P$, the proof of Corollary (3.4) only requires the hypothesis $E_{\left(\begin{array}{l}\mu-1 \\ 2\end{array}\right)}(\Lambda) \not \equiv 0$ (modulo char $P$ ). The same is true of

Corollary (3.5). If $E_{\left(\mu_{2}^{-1}\right)}(\Lambda)=\mathbf{Z}$, then

$$
\begin{aligned}
& \operatorname{in}_{s}\left(\left(\mathrm{id} \otimes \zeta_{3}\right)\left(P \otimes_{\mathrm{z}} Z_{3}\right)\right) \cap \operatorname{in}_{s}\left(\left(\mathrm{id} \otimes \lambda_{\mu}\right)\left(P \otimes_{\mathrm{z}} Y_{\mu}\right)\right) \\
& \quad=0 \subseteq \operatorname{gr}_{s}\left(P \otimes_{\mathrm{z}} Z_{2}\right)
\end{aligned}
$$

for every $s \geq 0$.

Proof. If $s=0$, in $\left(\left(\mathrm{id} \otimes \zeta_{3}\right)\left(P \otimes_{\mathrm{z}} Z_{3}\right)\right)=0$.

If $s \geq 1$, recall that by Lemma (2.3)

$$
\begin{aligned}
\text { in }_{s}\left(\left(\mathrm{id} \otimes \zeta_{3}\right)\left(P \otimes_{\mathrm{z}} Z_{3}\right)\right) \\
=\left(\mathrm{id} \otimes \zeta_{3}\right)\left(H_{s-1}\left(P \otimes_{\mathrm{z}} Z_{3}\right)\right) \\
\quad+(I H)^{s+1} \cdot\left(P \otimes_{\mathrm{z}} Z_{2}\right) /(I H)^{s+1} \cdot\left(P \otimes_{\mathrm{z}} Z_{2}\right)
\end{aligned}
$$


and

$$
\begin{aligned}
\operatorname{in}_{s}\left(\left(\mathrm{id} \otimes \lambda_{\mu}\right)\left(P \otimes_{\mathrm{z}} Y_{\mu}\right)\right) \\
=\left(\mathrm{id} \otimes \lambda_{\mu}\right)\left(H_{s}\left(P \otimes_{\mathrm{z}} Y_{\mu}\right)\right) \\
\quad+(I H)^{s+1} \cdot\left(P \otimes_{\mathrm{z}} Z_{2}\right) /(I H)^{s+1} \cdot\left(P \otimes_{\mathrm{z}} Z_{2}\right) .
\end{aligned}
$$

Suppose $z \in H_{s-1}\left(P \otimes_{\mathrm{z}} Z_{3}\right)$ and $y \in H_{s}\left(P \otimes_{\mathrm{Z}} Y_{\mu}\right)$ have

$$
\operatorname{in}\left(\left(\mathrm{id} \otimes \zeta_{3}\right)(z)\right)=\operatorname{in}\left(\left(\mathrm{id} \otimes \lambda_{\mu}\right)(y)\right) .
$$

Since $\left(\mathrm{id} \otimes \zeta_{3}\right)(z)$ and (id $\left.\otimes \lambda_{\mu}\right)(y)$ are homogeneous elements of $P \otimes_{\mathrm{z}} Z_{2}$, it follows from Proposition (2.1) that $\left(\right.$ id $\left.\otimes \zeta_{3}\right)(z)=$ $\left(\mathrm{id} \otimes \lambda_{\mu}\right)(y)$, and hence that $\left(\mathrm{id} \otimes\left(\zeta_{2} \lambda_{\mu}\right)\right)(y)=0$. By Corollary (3.4), then, $y=0$.

Recall that $B_{\Lambda}$ is the cokernel of the map $\left\langle\lambda_{\mu}, \zeta_{3}\right\rangle: Y_{\mu} \oplus Z_{3} \rightarrow Z_{2}$.

Proposition (3.6). If $E_{\left(\mu_{2}^{-1}\right)}(\Lambda)=\mathbf{Z}$, then for every $P$ and every $s \geq 0$, $P \otimes \mathrm{Z}_{s} \operatorname{gr}_{s}\left(B_{\Lambda}\right)$ is a free $P$-module of rank $(s+1) \cdot\left(\begin{array}{c}\mu+s-1 \\ s+2\end{array}\right)$.

Proof. Suppose $s \geq 0$. Note that $\operatorname{gr}_{s}\left(B_{\Lambda}\right)$ is a finitely generated abelian group (it is isomorphic to $\operatorname{gr}_{s}\left(Z_{2}\right) /$ in $_{s}\left(\left\langle\lambda_{\mu}, \zeta_{3}\right\rangle\left(Y_{\mu} \oplus Z_{3}\right)\right)$ ), and so it suffices to verify the assertion for $P \neq \mathbf{Z}$.

Suppose, then, that $P \neq \mathrm{Z}$. By Proposition (2.6), $P \otimes_{\mathrm{Z}} \operatorname{gr}_{s}\left(B_{\Lambda}\right) \cong$ $\operatorname{gr}_{s}\left(P \otimes_{\mathrm{Z}} B_{\Lambda}\right)$. By the right exactness of tensor products, $P \otimes_{\mathrm{Z}} B_{\Lambda}$ is isomorphic to the cokernel of id $\otimes\left\langle\lambda_{\mu}, \zeta_{3}\right\rangle: P \otimes_{\mathrm{Z}}\left(Y_{\mu} \oplus Z_{3}\right) \rightarrow P \otimes_{\mathrm{Z}} Z_{2}$, so $P \otimes_{\mathrm{z}} \mathrm{gr}_{s}\left(B_{\Lambda}\right)$ is isomorphic to

$$
\mathrm{gr}_{s}\left(P \otimes_{\mathrm{z}} Z_{2}\right) / \text { in }_{s}\left(\left(\mathrm{id} \otimes\left\langle\lambda_{\mu}, \zeta_{3}\right\rangle\right)\left(P \otimes_{\mathrm{z}}\left(Y_{\mu} \oplus Z_{3}\right)\right)\right) \text {. }
$$

By Proposition (2.4),

$$
\begin{aligned}
\operatorname{in}_{s}\left(\left(\mathrm{id} \otimes\left\langle\lambda_{\mu}, \zeta_{3}\right\rangle\right)\right)\left(P \otimes_{\mathrm{Z}}\left(Y_{\mu} \oplus Z_{3}\right)\right) \\
=\left(\mathrm{id} \otimes \lambda_{\mu}\right)\left(H_{s}\left(P \otimes_{\mathrm{Z}} Y_{\mu}\right)\right)+\left(\mathrm{id} \otimes \zeta_{3}\right)\left(H_{s-1}\left(P \otimes_{\mathrm{Z}} Z_{3}\right)\right) \\
\quad+(I H)^{s+1} \cdot\left(P \otimes_{\mathrm{Z}} Z_{2}\right) /(I H)^{s+1} \cdot\left(P \otimes_{\mathrm{Z}} Z_{2}\right) \\
=\text { in }_{s}\left(\left(\mathrm{id} \otimes \lambda_{\mu}\right)\left(P \otimes_{\mathrm{Z}} Y_{\mu}\right)\right)+\text { in }_{s}\left(\left(\mathrm{id} \otimes \zeta_{3}\right)\left(P \otimes_{\mathrm{Z}} Z_{3}\right)\right),
\end{aligned}
$$

and by Corollary (3.5), the latter sum is direct. Thus $P \otimes_{\mathrm{Z}} \mathrm{gr}_{s}\left(B_{\Lambda}\right)$ is a vector space of dimension $d-d_{1}-d_{2}$ over $P$, where $d, d_{1}$, and $d_{2}$ are the dimensions of $\operatorname{gr}_{s}\left(P \otimes_{\mathrm{z}} Z_{2}\right)$, in $s\left(\left(\mathrm{id} \otimes \lambda_{\mu}\right)\left(P \otimes_{\mathrm{z}} Y_{\mu}\right)\right)$, and in $_{s}\left(\left(\right.\right.$ id $\left.\left.\otimes \zeta_{3}\right)\left(P \otimes Z_{\mathrm{z}} Z_{3}\right)\right)$.

By Corollary $(2.2), d=\left(\begin{array}{c}\mu \\ 2\end{array}\right) \cdot\left(\begin{array}{c}\mu+s-1 \\ s\end{array}\right)$. 
Since the sum of the rows of $\Lambda$ is 0 , its elementary ideals are unchanged by the removal of any one of its rows. The matrix representing $\lambda_{\mu}$ (that is, the matrix obtained from $\Lambda$ by removing its last row) must then be a matrix of integers with $E_{\left(\mu_{2}^{\mu-1}\right)}=\mathbf{Z}$, so considering it as a matrix with entries from $\mathbf{Z} H$, it has $E_{\left(\mu^{-1}\right)}=\mathbf{Z} H$. By Lemma (2.7), then, $\lambda_{\mu}$ is a split monomorphism of $\mathbf{Z} H$-modules, and hence id $\otimes \lambda_{\mu}$ is a split monomorphism of $P H$-modules. It follows that $\operatorname{gr}\left(\mathrm{id} \otimes \lambda_{\mu}\right): \operatorname{gr}\left(P \otimes_{\mathrm{Z}} Y_{\mu}\right) \rightarrow$ $\operatorname{gr}\left(P \otimes_{\mathrm{z}} Z_{2}\right)$ is a split monomorphism of graded $\operatorname{gr}(P H)$-modules, so $\operatorname{gr}_{s}\left(\mathrm{id} \otimes \lambda_{\mu}\right)\left(\operatorname{gr}_{s}\left(P \otimes_{\mathrm{z}} Y_{\mu}\right)\right) \cong \operatorname{gr}_{s}\left(P \otimes_{\mathrm{Z}} Y_{\mu}\right)$ is a vector space of dimension $(\mu-1)\left(\begin{array}{c}\mu+s-1 \\ s\end{array}\right)$ over $P$. By Lemma (2.3),

$$
\begin{aligned}
\operatorname{in}_{s}\left(\left(\mathrm{id} \otimes \lambda_{\mu}\right)\left(P \otimes_{\mathrm{Z}} Y_{\mu}\right)\right) \\
=\left(\mathrm{id} \otimes \lambda_{\mu}\right)\left(H_{s}\left(P \otimes_{\mathrm{Z}} Y_{\mu}\right)\right) \\
\quad+(I H)^{s+1} \cdot\left(P \otimes_{\mathrm{Z}} Z_{2}\right) /(I H)^{s+1} \cdot\left(P \otimes_{\mathrm{Z}} Z_{2}\right) \\
=\mathrm{gr}_{s}\left(\mathrm{id} \otimes \lambda_{\mu}\right)\left(\mathrm{gr}_{s}\left(P \otimes_{\mathrm{Z}} Y_{\mu}\right)\right),
\end{aligned}
$$

so $d_{1}=(\mu-1) \cdot\left(\begin{array}{c}\mu+s-1 \\ s\end{array}\right)$.

By Proposition (2.5), $\operatorname{gr}_{s}\left(\operatorname{coker} \zeta_{3}\right) \cong \operatorname{gr}_{s}\left(Z_{2}\right) / \operatorname{in}_{s}\left(\zeta_{3}\left(Z_{3}\right)\right)$ is a free abelian group of rank $d-\left(\begin{array}{c}s+1 \\ 2\end{array}\right) \cdot\left(\begin{array}{c}\mu+s-1 \\ s+2\end{array}\right)$. By Proposition (2.6),

$$
\operatorname{gr}_{s}\left(\operatorname{coker}\left(\mathrm{id} \otimes \zeta_{3}\right)\right) \cong \operatorname{gr}_{s}\left(P \otimes_{\mathrm{z}} Z_{2}\right) / \mathrm{in}_{s}\left(\left(\mathrm{id} \otimes \zeta_{3}\right)\left(P \otimes_{\mathrm{z}} Z_{3}\right)\right)
$$

must then be a vector space over $P$ of dimension $d-\left(\begin{array}{c}s+1 \\ 2\end{array}\right) \cdot\left(\begin{array}{c}\mu+s-1 \\ s+2\end{array}\right)$; necessarily then $d_{2}=\left(\begin{array}{c}s+1 \\ 2\end{array}\right) \cdot\left(\begin{array}{c}\mu+s-1 \\ s+2\end{array}\right)$.

Thus $P \otimes_{\mathrm{Z}} \mathrm{gr}_{s}\left(B_{\Lambda}\right)$ is a vector space over $P$ of dimension

$$
\begin{aligned}
d- & d_{1}-d_{2} \\
= & \left(\begin{array}{c}
\mu \\
2
\end{array}\right) \cdot\left(\begin{array}{c}
\mu+s-1 \\
s
\end{array}\right)-(\mu-1) \cdot\left(\begin{array}{c}
\mu+s-1 \\
s
\end{array}\right) \\
& -\left(\begin{array}{c}
s+1 \\
2
\end{array}\right) \cdot\left(\begin{array}{c}
\mu+s-1 \\
s+2
\end{array}\right) \\
= & \left(\begin{array}{c}
\mu+s-1 \\
s
\end{array}\right) \cdot\left[\left(\begin{array}{c}
\mu-1 \\
2
\end{array}\right)-\frac{(\mu-2)(\mu-1)}{(s+1)(s+2)} \cdot\left(\begin{array}{c}
s+1 \\
2
\end{array}\right)\right] \\
= & \left(\begin{array}{c}
\mu+s-1 \\
s
\end{array}\right) \cdot\left(\begin{array}{c}
\mu-1 \\
2
\end{array}\right) \cdot\left(1-\frac{s}{s+2}\right) \\
= & \left(\begin{array}{c}
\mu+s-1 \\
s
\end{array}\right) \cdot \frac{(\mu-1)(\mu-2)}{s+2}=\left(\begin{array}{c}
\mu+s-1 \\
s+2
\end{array}\right) \cdot(s+1) .
\end{aligned}
$$

Recalling Proposition (3.2), we conclude that if $F$ is a free group on $\mu-1$ generators then condition (b) of Theorem 1 implies that $\operatorname{gr}_{s}\left(B_{\Lambda}\right) \cong$ $F^{\prime \prime} F_{s+2} / F^{\prime \prime} F_{s+3} \forall s \geq 0$. Since $G^{\prime \prime} G_{q} / G^{\prime \prime} G_{q+1}=\operatorname{gr}_{q-2}\left(B_{L}\right) \cong \operatorname{gr}_{q-2}\left(\hat{B}_{L}\right)$ 
for $q \geq 2$ (as we noted at the beginning of this section), to complete the proof of $(b) \Rightarrow$ (c) it suffices to show that when (b) holds, $\operatorname{gr}\left(B_{\Lambda}\right) \cong \operatorname{gr}\left(\hat{B}_{L}\right)$. This isomorphism actually exists under a slightly weaker hypothesis, as we shall see in Proposition (3.8).

LEMMA (3.7). If $E_{\left(\mu_{2}^{-1}\right)}(\Lambda) \neq 0$, then

$$
\operatorname{in}\left(\nu_{\mu}\left(\hat{Y}_{\mu}\right)\right)=\operatorname{in}\left(\lambda_{\mu}\left(Y_{\mu}\right)\right) \subseteq \operatorname{gr}\left(Z_{2}\right)=\operatorname{gr}\left(\hat{Z}_{2}\right) .
$$

Proof. Note that we are identifying $\operatorname{gr}\left(Z_{2}\right)$ and $\operatorname{gr}\left(\hat{Z}_{2}\right)$ via the isomorphism induced by the inclusion $Z_{2} \subseteq \hat{Z}_{2}$.

By Lemma (2.3),

$$
\operatorname{in}_{s}\left(\lambda_{\mu}\left(Y_{\mu}\right)\right)=\lambda_{\mu}\left(H_{s}\left(Y_{\mu}\right)\right)+(I H)^{s+1} \cdot Z_{2} /(I H)^{s+1} \cdot Z_{2}
$$

for every $s \geq 0$. Since $\nu_{\mu}(y) \equiv \hat{\lambda}_{\mu}(y)$ (modulo $\left.\widehat{I H} \cdot \hat{Z}_{2}\right) \forall y \in \hat{Y}_{\mu}$, certainly $\nu_{\mu}(h) \equiv \lambda_{\mu}(h)$ (modulo $(\overline{I H})^{s+1} \cdot \hat{Z}_{2}$ ) for all $h \in H_{s}\left(Y_{\mu}\right)$ for every $s \geq 0$, and so in ${ }_{s}\left(\lambda_{\mu}\left(Y_{\mu}\right)\right) \subseteq \operatorname{in}_{s}\left(\nu_{\mu}\left(\hat{Y}_{\mu}\right)\right)$ for every $s \geq 0$.

On the other hand, suppose $y=\sum a_{i} x_{i} \in \hat{Y}_{\mu}$ and $\nu_{\mu}(y) \in(\widehat{I H})^{s} \cdot \hat{Z}_{2}$; let $s-t=\min \left\{v\left(a_{\imath}\right)\right\}$. If $t=0$, then $\operatorname{in}\left(\nu_{\mu}(y)\right)=\operatorname{in}\left(\hat{\lambda}_{\mu}(y)\right) \in$ in $_{s}\left(\lambda_{\mu}\left(Y_{\mu}\right)\right)$. Suppose instead that $t>0$. For each $i<\mu$, let $h_{i} \in$ $H_{s-t}(\mathbf{Z} H)$ be the homogeneous element with $\operatorname{in}\left(h_{i}\right)=\operatorname{in}\left(a_{t}\right)$ (if $v\left(a_{i}\right)=s$ $-t$ ) or $h_{i}=0$ (if $v\left(a_{i}\right)>s-t$ ). Then $a_{i}-h_{i} \in(\widehat{I H})^{s-t+1} \forall i$, so $\nu_{\mu}\left(\Sigma\left(h_{l}-a_{i}\right) x_{i}\right) \in\left(\widehat{I H}^{s-t+1} \cdot \hat{Z}_{2} ;\right.$ since $\nu_{\mu}(y) \in(\widehat{I H})^{s-t+1} \cdot \hat{Z}_{2}$, then, $\nu_{\mu}\left(\sum h_{l} x_{i}\right) \in\left(\widehat{I H}^{s-t+1} \cdot \hat{Z}_{2}\right.$. Since $\nu_{\mu}\left(x_{l}\right) \equiv \lambda_{\mu}\left(x_{i}\right)$ (modulo $\left.\overparen{I H} \cdot \hat{Z}_{2}\right) \forall i$, it follows that $\lambda_{\mu}\left(\sum h_{i} x_{i}\right) \in(\widehat{I H})^{s-t+1} \cdot \hat{Z}_{2}$. But $\lambda_{\mu}\left(\sum h_{i} x_{i}\right)$ is homogeneous of degree $s-t$, so $\lambda_{\mu}\left(\sum h_{i} x_{l}\right)=0$, by Proposition (2.1). By Corollary (3.4), applied with $P=\mathbf{Z}$ (see the comment preceding Corollary (3.5)), $\lambda_{\mu}$ is injective; necessarily then $h_{l}=0 \forall i$. This is impossible, though, for by the definition of $t, v\left(a_{i}\right)=v\left(h_{i}\right)=s-t<\infty$ for some $i$. Thus it must be that $t=0$.

Proposition (3.8). If $E_{\left(\mu_{2}^{\mu-1}\right)}(\Lambda) \neq 0$, then

$$
\begin{aligned}
\operatorname{in}\left(\left\langle\nu_{\mu}, \hat{\zeta}_{3}\right\rangle\left(\hat{Y}_{\mu} \oplus \hat{Z}_{3}\right)\right) & =\operatorname{in}\left(\left\langle\lambda_{\mu}, \zeta_{3}\right\rangle\left(Y_{\mu} \oplus Z_{3}\right)\right) \\
& \subseteq \operatorname{gr}\left(Z_{2}\right)=\operatorname{gr}\left(\hat{Z}_{2}\right),
\end{aligned}
$$

and consequently $\operatorname{gr}\left(\hat{B}_{L}\right) \cong \operatorname{gr}\left(B_{\Lambda}\right)$.

Proof. We assert that $\operatorname{in}\left(\left\langle\nu_{\mu}, \hat{\zeta}_{3}\right\rangle\left(\hat{Y}_{\mu} \oplus \hat{Z}_{3}\right)\right)=\operatorname{in}\left(\nu_{\mu}\left(\hat{Y}_{\mu}\right)\right)+\operatorname{in}\left(\hat{\zeta}_{3}\left(\hat{Z}_{3}\right)\right)$. Obviously the former contains the latter, so to verify this equality it 
suffices to show that whenever $y \in \hat{Y}_{\mu}$ and $z \in \hat{Z}_{3}$ have $v\left(\nu_{\mu}(y)+\hat{\zeta}_{3}(z)\right)$ $=s$, there are $\nu_{\mu}\left(y^{\prime}\right), \hat{\zeta}_{3}\left(z^{\prime}\right) \in(\widehat{I H})^{s} \cdot \hat{Z}_{2}$ with $\operatorname{in}\left(\nu_{\mu}(y)+\hat{\zeta}_{3}(z)\right)=$ $\operatorname{in}\left(\nu_{\mu}\left(y^{\prime}\right)+\hat{\zeta}_{3}\left(z^{\prime}\right)\right)$. If either of $\nu_{\mu}(y), \hat{\zeta}_{3}(z)$ is in $(\widehat{I H})^{s} \cdot \hat{Z}_{2}$, then both are, and we may take $y=y^{\prime}$ and $z=z^{\prime}$. If neither is, then we must have $v=v\left(\nu_{\mu}(y)\right)=v\left(\hat{\zeta}_{3}(z)\right)<s$ and $\operatorname{in}\left(\nu_{\mu}(y)\right)=-\operatorname{in}\left(\hat{\zeta}_{3}(z)\right)$. By Lemma (3.7), then, $\operatorname{in}\left(\hat{\zeta}_{3}(z)\right) \in \operatorname{in}_{v}\left(\lambda_{\mu}\left(Y_{\mu}\right)\right)$. By Corollary (3.5) (with $P=Z$ ), this implies that $\operatorname{in}\left(\hat{\zeta}_{3}(z)\right)=0$, an impossibility since $v\left(\hat{\zeta}_{3}(z)\right)<\infty$.

Having verified our assertion, we may cite Lemma (2.3), Proposition (2.4), and Lemma (3.7) to verify that for every $s \geq 0$,

$$
\begin{aligned}
\operatorname{in}_{s}\left(\left\langle\nu_{\mu}, \hat{\zeta}_{3}\right\rangle\right. & \left.\left(\hat{Y}_{\mu} \oplus \hat{Z}_{3}\right)\right)=\operatorname{in}_{s}\left(\nu_{\mu}\left(\hat{Y}_{\mu}\right)\right)+\operatorname{in}_{s}\left(\hat{\zeta}_{3}\left(\hat{Z}_{3}\right)\right) \\
& =\operatorname{in}_{s}\left(\lambda_{\mu}\left(Y_{\mu}\right)\right)+\operatorname{in}_{s}\left(\zeta_{3}\left(Z_{3}\right)\right) \\
& =\lambda_{\mu}\left(H_{s}\left(Y_{\mu}\right)\right)+\zeta_{3}\left(H_{s-1}\left(Z_{3}\right)\right)+(I H)^{s+1} \cdot Z_{2} /(I H)^{s+1} \cdot Z_{2} \\
& =\operatorname{in}_{s}\left(\left\langle\lambda_{\mu}, \zeta_{3}\right\rangle\left(Y_{\mu} \oplus Z_{3}\right)\right) .
\end{aligned}
$$

The isomorphism of the statement follows immediately, since

$$
\begin{gathered}
\operatorname{gr}\left(\hat{B}_{L}\right) \cong \operatorname{gr}\left(\hat{Z}_{2}\right) / \operatorname{in}\left(\left\langle\nu_{\mu}, \hat{\zeta}_{3}\right\rangle\left(\hat{Y}_{\mu} \oplus \hat{Z}_{3}\right)\right) \text { and } \\
\operatorname{gr}\left(B_{\Lambda}\right) \cong \operatorname{gr}\left(Z_{2}\right) / \operatorname{in}\left(\left\langle\lambda_{\mu}, \zeta_{3}\right\rangle\left(Y_{\mu} \oplus Z_{3}\right)\right) .
\end{gathered}
$$

Proposition (3.8) completes our proof of the fact that condition (b) of Theorem 1 implies condition (c). As we noted in the introduction, the implication (a) $\Rightarrow$ (b) and the equivalence (c) $\Leftrightarrow$ (d) follow from the work of K.-T. Chen [3] and T. Maeda [8]; since (a) is simply a special case of (d), we may now conclude that each of (a), (b), (c), and (d) is equivalent to any other.

Before proceeding to consider (e), we present another condition equivalent to those of Theorem 1 . To motivate this condition, note that by [16, Corollary (4.5)] the completion $\hat{B}_{L}$ of the Alexander invariant has the property that its elementary ideal $E_{\left(\mu_{2}^{-1}\right)-1}\left(\hat{B}_{L}\right)$ is contained in $\widehat{I H}$. Since a $\widehat{\mathrm{ZH}}$-module generated by some $k$ of its elements must have $E_{k}=\widehat{\mathbf{Z H}}[12$, Theorem 3.2], this implies that no set of fewer than $\left(\begin{array}{c}\mu-1 \\ 2\end{array}\right)$ elements could possibly generate $\hat{B}_{L}$.

THEOREM (3.9). The conditions of Theorem 1 hold if, and only if, $\hat{B}_{L}$ can be generated (as a $\widehat{\mathrm{ZH}}$-module) by some $\left(\begin{array}{c}\mu-1 \\ 2\end{array}\right)$ of its elements.

Proof. (We actually prove that this condition is equivalent to (b).) 
First, suppose that $\hat{B}_{L}$ can be generated by some $\left({ }_{2}^{\mu-1}\right)$ of its elements. Then $E_{\left(\mu_{2}^{-1}\right)}\left(\hat{B}_{L}\right)=\widehat{\mathbf{Z H}}$, so certainly $\varepsilon E_{\left(\mu^{-1}\right)}\left(\hat{B}_{L}\right)=\mathbf{Z}$. Let $\mathcal{N}$ be the matrix representing the map $\nu_{0}: \hat{Y}_{0} \rightarrow \hat{Z}_{2}^{2}$ that appears in the exact sequence (3.1). Then by [16, Theorem (4.4)] (with $q=1$ ),

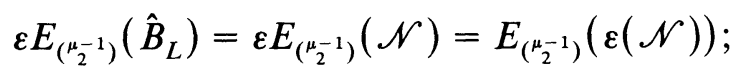

as $\varepsilon(\mathscr{N})$ is precisely the matrix $\Lambda$, this shows that (b) holds.

Conversely, suppose (b) holds. Let $\Lambda_{\mu}$ and $\mathscr{N}_{\mu}$ be the matrices obtained from $\Lambda$ and $\mathscr{N}$ by deleting the last row of each. Since the sum of the rows of $\Lambda$ is zero, (b) implies that $E_{\left(\mu_{2}^{-1}\right)}\left(\Lambda_{\mu}\right)=\mathbf{Z}$ too. Since $\varepsilon(\mathscr{N})=\Lambda, \varepsilon\left(\mathscr{N}_{\mu}\right)=\Lambda_{\mu}$, and so $\varepsilon E_{\left({ }^{\mu}-1\right)}\left(\mathscr{N}_{\mu}\right)=\mathbf{Z}$; thus $E_{\left({ }^{\mu}-1\right)}\left(\mathscr{N}_{\mu}\right)$ is an ideal of $\widehat{\mathbf{Z H}}$ which contains some element whose image under $\varepsilon$ is 1 . If we identify $\widehat{\mathbf{Z H}}$ with the power series ring $\mathbf{Z}\left[\left[u_{1}, \ldots, u_{\mu}\right]\right]$ (as discussed in §2), this element will be a power series whose constant term is 1; a well-known property of the power series ring is that such an element must be a unit. Thus $E_{\left({ }^{\mu-1}\right)}\left(\mathscr{N}_{\mu}\right)$ must be all of $\widehat{\mathbf{Z H}}$, so by Lemma (2.7), the map $\nu_{\mu}$ : $\hat{Y}_{\mu} \rightarrow \hat{Z}_{2}$ must be a split monomorphism, since it is represented by the matrix $\mathscr{N}_{\mu}$. It follows immediately from this and the exactness of (3.1) that $\hat{B}_{L}$ can be generated by $\left(\begin{array}{c}\mu \\ 2\end{array}\right)-(\mu-1)=\left(\begin{array}{c}\mu-1 \\ 2\end{array}\right)$ of its elements.

When $\mu$ is two or three and the equivalent conditions of Theorem 1 hold, this additional condition allows us to determine the structure of the $\widehat{\mathbf{Z H}}$-module $\hat{B}_{L}$. If $\mu=2, \hat{B}_{L}$ can be generated by $\left({ }_{2}^{1}\right)=0$ of its elements; that is, $\hat{B}_{L}=0$. If $\mu=3, \hat{B}_{L}$ can be generated by $\left(\begin{array}{l}2 \\ 2\end{array}\right)=1$ element, so it is isomorphic to $\widehat{\mathrm{ZH}} / E_{0}\left(\hat{B}_{L}\right)$ [12, Theorem 3.5]; by [16, Theorem (4.1)], $E_{0}\left(\hat{B}_{L}\right)$ is the principal ideal of $\widehat{\mathbf{Z H}}$ generated by the Alexander polynomial of $L$.

4. Proof that (e) $\Leftrightarrow$ (a). We will re-phrase the assertion that statements (e) and (a) of Theorem 1 are equivalent as follows:

Proposition (4.1). Let $L$ be a tame link of $\mu$ components in $S^{3}$ with group $G$ and complementary space $X=S^{3}-L$. Then $G_{2} / G_{3}$ is a free abelian group of rank $\left({ }_{2}^{\mu-1}\right)$ if and only if the cup product pairing (with integer coefficients)

$$
H^{1}(X) \otimes_{\mathrm{z}} H^{1}(X) \rightarrow H^{2}(X)
$$

is epimorphic. 
Proof. First of all, we assert that if $G_{2} / G_{3}$ is free abelian of rank $\left(\begin{array}{c}\mu-1 \\ 2\end{array}\right)$, then the link $L$ is unsplittable. For, it is readily verified that if the link $L$ is splittable, then $E_{\left(\mu_{2}-1\right)}(\Lambda)=0$. Since conditions (a) and (b) are equivalent, the assertion follows.

Similarly, we assert that if the cup product pairing (4.1) is epimorphic, then the link $L$ is unsplittable. To prove this assertion, one proves that if the link $L$ is splittable, then the image of the cup product pairing (4.1) is a subgroup of $H^{2}(X)$ which has rank at most $\mu-2$ (the proof uses the Mayer-Vietoris cohomology exact sequence, the naturality of cup products, and the fact that (by Alexander duality) $H^{2}(X)$ is free abelian of rank $\mu-1)$.

In view of these two assertions, we see that we may assume that the link $L$ is unsplittable. As a consequence, the complementary space $X$ is an Eilenberg-MacLane complex, $K(G, 1)\left[13\right.$, p. 19], and $H_{i}(X)=H_{i}(G)$ for all $i$.

To prove the proposition, we will use the following five-term exact sequence:

$$
H_{2}(G) \stackrel{p *}{\rightarrow} H_{2}\left(G / G_{2}\right) \rightarrow G_{2} / G_{3} \rightarrow H_{1}(G) \stackrel{1}{\rightarrow} H_{1}\left(G / G_{2}\right) \rightarrow 0
$$

(cf. p. 205 of [4]). In this exact sequence, arrow number 1 is obviously an isomorphism, hence the sequence simplifies to the following:

$$
H_{2}(G) \stackrel{p *}{\rightarrow} H_{2}\left(G / G_{2}\right) \rightarrow G_{2} / G_{3} \rightarrow 0 .
$$

Now $G / G_{2}$ is a free abelian group of rank $\mu$, hence

$$
K\left(G / G_{2}, 1\right)=\left(S^{1}\right)^{\mu}
$$

the cartesian product of $\mu$ copies of the circle $S^{1}$. Therefore $H_{2}\left(G / G_{2}\right)$ is a free abelian group of rank $\left(\begin{array}{c}\mu \\ 2\end{array}\right)$. Since $H_{2}(G)=H_{2}(X)$ is free abelian of rank $\mu-1$, we see that $G_{2} / G_{3}$ is free abelian of rank $\left(\begin{array}{c}\mu-1 \\ 2\end{array}\right)$ if and only if $p_{*}$ is a split monomorphism. Next, recall that the integral homology groups of $G$ and $G / G_{2}$ are all free abelian of finite rank. From this and the universal coefficient theorem [4, p. 222] it follows that $p_{*}$ is a split monomorphism if and only if the dual homomorphism in integral cohomology,

$$
p^{*}: H^{2}\left(G / G_{2}\right) \rightarrow H^{2}(G),
$$

is a split epimorphism. Now observe that

$$
p^{*}: H^{1}\left(G / G_{2}\right) \rightarrow H^{1}(G)
$$


is an isomorphism, and that the following diagram is commutative:

$$
\begin{array}{ccc}
H^{1}\left(G / G_{2}\right) \otimes_{\mathrm{z}} H^{1}\left(G / G_{2}\right) & \stackrel{\cup}{\rightarrow} & H^{2}\left(G / G_{2}\right) \\
\downarrow & & \downarrow p^{*} \otimes p^{*} \\
H^{1}(G) \otimes_{\mathrm{z}} H^{1}(G) & \stackrel{\cup}{\rightarrow} & H^{2}(G)
\end{array}
$$

(the horizontal arrows denote cup product homomorphisms). Since the cup product in the top line of this diagram is epimorphic (this follows readily from a cohomological version of the Künneth theorem [10, $\S$ VIII.11]), we conclude that

$$
p^{*}: H^{2}\left(G / G_{2}\right) \rightarrow H^{2}(G)
$$

is epimorphic if and only if the cup product in the bottom line is epimorphic. This completes the proof.

5. The Hosokawa polynomial. Let $L$ be a tame link of $\mu$ components in $S^{3}$, and $l$ the $\mu \times \mu$ matrix with entries given by $l_{i i}=-\sum_{k \neq i} l\left(K_{l}, K_{k}\right)$, and for $i \neq j, l_{i j}=l\left(K_{i}, K_{j}\right)$. Then $l=\Lambda M$ for an appropriate $\left(\begin{array}{l}\mu \\ 2\end{array}\right) \times \mu$ integral matrix $M$, and so (by the functoriality of the exterior algebra), the determinant of a square submatrix of $l$ can be expressed as an integral linear combination of the determinants of the various square submatrices of $\Lambda$ of the same size; that is, $E_{\mu-m}(l) \subseteq E_{\left(\frac{\mu}{2}\right)-m}(\Lambda) \forall m$.

In particular, $E_{1}(l) \subseteq E_{\left(\mu_{2}^{-1}\right)}(\Lambda)$. The Hosokawa polynomial $\nabla(L)$ has the property that $\nabla(L)(1)$ generates the ideal $E_{1}(l)$; in fact, every $(\mu-1) \times(\mu-1)$ submatrix of $l$ has determinant $\pm \nabla(L)(1)$ [5]. Thus we conclude

Proposition (5.1). $\nabla(L)(1) \in E_{\left(\mu_{2}^{-1}\right)}(\Lambda)$.

Consequently, if $\nabla(L)(1)= \pm 1$ then $E_{\left(\mu_{2}^{-1}\right)}(\Lambda)=\mathbf{Z}$.

\section{REFERENCES}

[1] N. Bourbaki, Commutative Algebra, Hermann, Paris and Addison-Wesley, Reading, Mass., 1972.

[2] K.-T. Chen, Integration in free groups, Ann. Math., (2) 54 (1951), 147-162.

[3] _ Commutator calculus and link invariants, Proc. Amer. Math. Soc., 3 (1952), 44-55, 993.

[4] P. J. Hilton and U. Stammbach, A Course in Homological Algebra, Springer-Verlag, Berlin and New York, 1971.

[5] F. Hosokawa, On $\nabla$-polynomials of links, Osaka Math. J., 10 (1958), 273-282. 
[6] S. Kojima, Nilpotent completions and Lie rings associated to link groups, Comment. Math. Helv., 58 (1983), 115-134.

[7] S. MacLane, Homology, Springer-Verlag, Berlin and New York, 1967.

[8] T. Maeda, Lower central series of link groups, Ph.D. dissertation, Univ. of Toronto, 1983.

[9] W. S. Massey, Completion of link modules, Duke Math. J., 47 (1980), 399-420.

[10] Singular Homology Theory, Springer-Verlag, Berlin and New York, 1980.

[11] K. Murasugi, On Milnor's invariant for links. II. The Chen group, Trans. Amer. Math. Soc., 148 (1970), 41-61.

[12] D. G. Northcott, Finite Free Resolutions, Cambridge Univ. Press, Cambridge, 1976.

[13] C. D. Papakyriakopoulos, On Dehn's lemma and the asphericity of knots, Ann. Math., (2) 66 (1957), 1-26.

[14] J. Stallings, Homology and central series of groups, J. Algebra, 2 (1965), 170-181.

[15] G. Torres, On the Alexander polynomial, Ann. Math., (2) 57 (1953), 57-89.

[16] L. Traldi, Milnor's invariants and the completions of link modules, Trans. Amer. Math. Soc., 284 (1984), 401-424.

[17] O. Zariski and P. Samuel, Commutative Algebra, Vol. II, Van Nostrand, Princeton, N.J., 1960.

Received March 10, 1985.

YALE UNIVERSITY

New HaVen, CT 06520

AND

Lafa yetTe College

EASTON, PA 18042 



\section{PACIFIC JOURNAL OF MATHEMATICS EDITORS}

\author{
V. S. VARADARAJAN \\ (Managing Editor) \\ University of California \\ Los Angeles, CA 90024 \\ HeRbert Clemens \\ University of Utah \\ Salt Lake City, UT 84112 \\ R. FINN \\ Stanford University \\ Stanford, CA 94305
}

HERMANN FLASCHKA

University of Arizona

Tucson, AZ 85721

RAMESH A. GANGOLLI

University of Washington

Seattle, WA 98195

VAUGHAN F. R. JONES

University of California

Berkeley, CA 94720

ROBION KIRBY

University of California

Berkeley, CA 94720
C. C. MOORE

University of California

Berkeley, CA 94720

H. SAMELSON

Stanford University

Stanford, CA 94305

HAROLD STARK

University of California, San Diego

La Jolla, CA 92093

\section{ASSOCIATE EDITORS}
R. Arens
E. F. BECKENBACH
B. H. NEUMANN
F. WOLF
K. YoshIDA (1906-1982)

\section{SUPPORTING INSTITUTIONS}

UNIVERSITY OF ARIZONA

UNIVERSITY OF BRITISH COLUMBIA

CALIFORNIA INSTITUTE OF TECHNOLOGY

UNIVERSITY OF CALIFORNIA

MONTANA STATE UNIVERSITY

UNIVERSITY OF NEVADA, RENO

NEW MEXICO STATE UNIVERSITY

OREGON STATE UNIVERSITY
UNIVERSITY OF OREGON

UNIVERSITY OF SOUTHERN CALIFORNIA

TANFORD UNIVERSITY

UNIVERSITY OF HAWAII

UNIVERSITY OF TOKYO

UNIVERSITY OF UTAH

WASHINGTON STATE UNIVERSITY

UNIVERSITY OF WASHINGTON 


\section{Pacific Journal of Mathematics}

Vol. 124, No. $1 \quad$ May, 1986

Kinetsu Abe and Martin Andrew Magid, Relative nullity foliations and indefinite isometric immersions

Erik P. van den Ban, A convexity theorem for semisimple symmetric

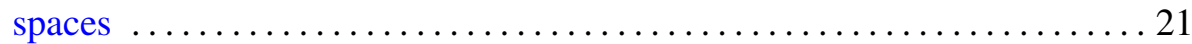

Bo Berndtsson and Thomas Joseph Ransford, Analytic multifunctions, the $\bar{\partial}$-equation, and a proof of the corona theorem .................. 57

Brian Boe and David H. Collingwood, Intertwining operators between

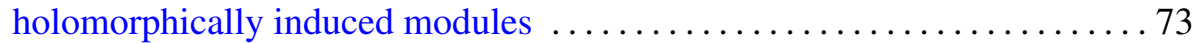

Giuseppe Ceresa and Alessandro Verra, The Abel-Jacobi isomorphism for

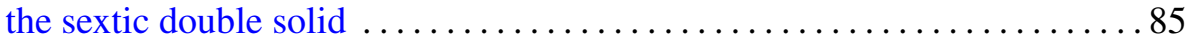

Kun Soo Chang, Jae Moon Ahn and Joo Sup Chang, An evaluation of the conditional Yeh-Wiener integral ........................... 107

Charles Dale Frohman, Minimal surfaces and Heegaard splittings of the

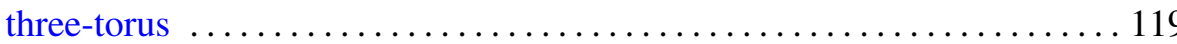

Robert M. Guralnick, Power cancellation of modules $\ldots \ldots \ldots \ldots \ldots \ldots 131$

Kenneth Hardy and Kenneth S. Williams, On the solvability of the Diophantine equation $d V^{2}-2 e V W-d W^{2}=1 \ldots \ldots \ldots \ldots \ldots \ldots \ldots \ldots \ldots$

Ray Alden Kunze and Stephen Scheinberg, Alternative algebras having

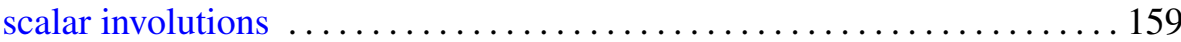

W. B. Raymond Lickorish and Kenneth Millett, The reversing result for the Jones polynomial $\ldots \ldots \ldots \ldots \ldots \ldots \ldots \ldots \ldots$

Guido Lupacciolu, A theorem on holomorphic extension of CR-functions

William Schumacher Massey and Lorenzo Traldi, On a conjecture of K. Murasugi

Dinakar Ramakrishnan, Spectral decomposition of $L^{2}(N \backslash \mathrm{GL}(2), \eta)$

Steven L. Sperber, On solutions of differential equations which satisfy certain algebraic relations 